\title{
Fluorescence spectroscopy approach for blood influence compensation
}

Shupletsov, Valery, Dremin, Viktor, Zherebtsov, Evgeny , Mezentsev, Mikhail, Kozlov, Igor, et al.

Valery Shupletsov, Viktor Dremin, Evgeny Zherebtsov, Mikhail Mezentsev, Igor Kozlov, Elena Potapova, Andrey Dunaev, "Fluorescence spectroscopy approach for blood influence compensation," Proc. SPIE 11065, Saratov Fall Meeting 2018: Optical and Nano-Technologies for Biology and Medicine, 110650R (3 June 2019); doi: 10.1117/12.2522794

SDIE Event: International Symposium on Optics and Biophotonics VI: Saratov Fall Meeting 2018, 2018, Saratov, Russian Federation 


\title{
Fluorescence spectroscopy approach for blood influence compensation
}

\author{
Valery Shupletsov ${ }^{\mathrm{a} *}$, Viktor Dremin $^{\mathrm{a}}$, Evgeny Zherebtsov ${ }^{\mathrm{a}, \mathrm{b}}$, Mikhail Mezentsev ${ }^{\mathrm{a}}$, Igor Kozlov ${ }^{\mathrm{a}}$, \\ Elena Potapova ${ }^{\mathrm{a}}$, Andrey Dunaev ${ }^{\mathrm{a}}$ \\ ${ }^{a}$ Research and Development Center of Biomedical Photonics, Orel State University \\ named after I.S. Turgenev, Orel, Russia; \\ ${ }^{b}$ Optoelectronics and Measurement Techniques, University of Oulu, Oulu, Finland
}

\begin{abstract}
At present, fluorescence spectroscopy (FS) and diffuse reflectance spectroscopy (DRS) are widespread methods highly used in medical practice. The combined application of these methods is a promising tool to improve the predictive force of classifiers for tissue type recognition as well as to compensate the attenuation of the fluorescence radiation by blood for the accurate evaluation of the biomarkers content in living tissue. Several techniques are known to normalize the resulting fluorescence spectrum in order to exclude the attenuation effect. In this study, an approach based on the dividing of the experimentally obtained FS spectrum by DRS spectrum has been applied for experiments with occlusion test. The implemented multimodal approach for the in vivo optical measurements in combination with occlusion test for minimisation of blood influence has shown good repeatability of obtained experimental fluorescence spectra. The results are of particular interest for the further development of methods for compensating the influence of chromophores in optical spectroscopy.
\end{abstract}

Keywords: fluorescence spectroscopy, diffuse reflectance spectroscopy, blood absorption, blood compensation.

\section{INTRODUCTION}

Currently, in various fields of biomedical engineering and medicine, a number of methods of optical spectroscopy are applied. One of these methods is the fluorescence spectroscopy using for the registration of emission from the endogenous fluorophores as well as fluorescence contrasts. ${ }^{1,2}$ In many in vivo applications of the method the attenuation of the intensity of fluorescence emission ${ }^{3,4}$ by blood is a challenging factor. The regions of interest for measurements in biological tissues in vivo contain a large number of different vessels (arterioles, capillaries, venules). Thus, the blood content in these vessels has a significant effect on fluorescent spectra ${ }^{5}$ due to the absorption and scattering in RBC. Also, absorption characteristics of blood depend on the level of the oxygen saturation. ${ }^{6}$ Blood volume fraction is a highly variable parameter, which directly affects the variability of the parameters, evaluated from fluorescence intensity. Therefore, it is essential to carry out the correction of the blood influence for most of in vivo applications of the FS measurements.

Up to date, there are a number of methods to compensate for the absorption of blood. ${ }^{7-11}$ In this study, an approach based on the dividing of the experimentally obtained FS spectrum by DRS spectrum has been applied for experiments with occlusion test. During the measurements DRS and FS data registered by the same fiber optical probe allowing for the simultaneous processing of the two different modalities to take into account the scattering and absorbing properties of the tissue under study. ${ }^{12}$

It is well known that the pressure applied on optical diagnostic probes is a significant factor affecting the results of measurements. ${ }^{13}$ Another example of a functional physiological test that has an prominent effect on the blood volume fraction in skin as well as state of vascular constriction is an occlusion test. During its practical implementation, the exceeding pressure is created and maintained for a few minutes in the occlusive cuff on the limb. When cuff vascular clamping is removed, typically, a sharp increase of perfusion and temperature are observed with the subsequent restoration to normal. In physiology, this fact is explained as a vasodilation of blood vessels due to the accumulation of metabolic vasodilators during occlusion. ${ }^{14}$

This work aims to test the blood content compensation technique in experiments with upper arm occlusion in healthy volunteers.

*email: matoka_97@mail.ru; phone:+79536111625; http://bmecenter.ru/en 


\section{THE METHOD OF RESEARCH}

The functional diagram of the experimental setup is shown in Fig. 1. FS channel has been composed of two emitting and one collecting fibers. DRS channel has been implemented with one emitting and one receiving fibers. Both channels were combined in a single fiber optical probe. The diagnostic volume and depth of sensing for this probe in the spectral area of hemoglobin absorption (540-580 nm) are equal to respectively $1.0-1.3 \mathrm{~mm}^{3}$ and $0.4-0.5 \mathrm{~mm} .{ }^{15}$ A UV light (LED) $365 \mathrm{~nm}$ and blue light sources (laser) $450 \mathrm{~nm}$ were used to excite the emission of fluorophores. A broadband tungsten halogen light source (HL-2000-HP-232R, "Ocean Optics", USA) was used in DRS channel. Collected light was analyzed by a CCD spectrometer (FLAME, "Ocean Optics", USA). A personal computer was used to control the system and automate the data acquisition process. The data processing has been performed using bespoke developed software in MATLAB program environment.

Tungsten halogen source HL-2000

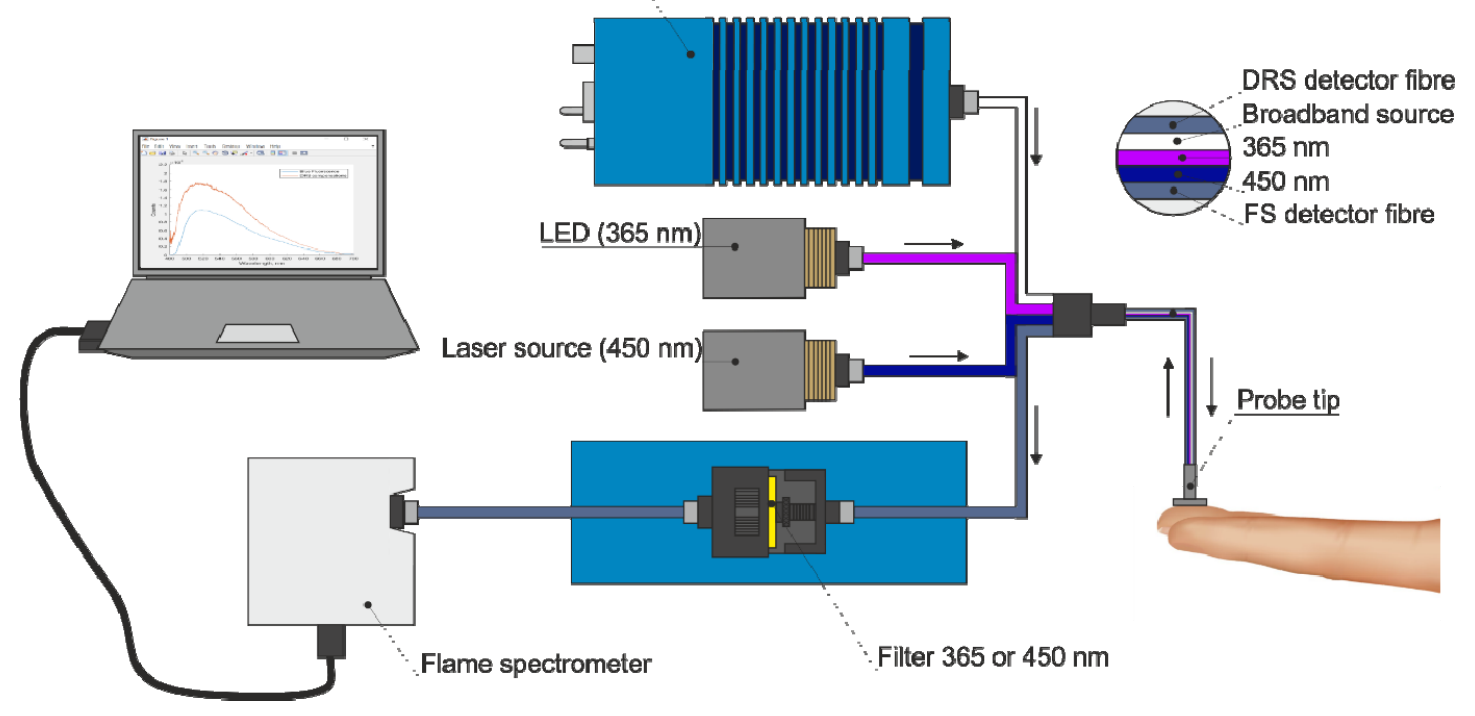

Figure 1. The experimental setup

The study involved 22 healthy volunteers (mean age was $22 \pm 3$ years. Experiments were conducted in a sitting position with hands placed on a table. The fiber optic probe was allocated on the palm surface of the distal phalange of the middle finger (Fig. 2).

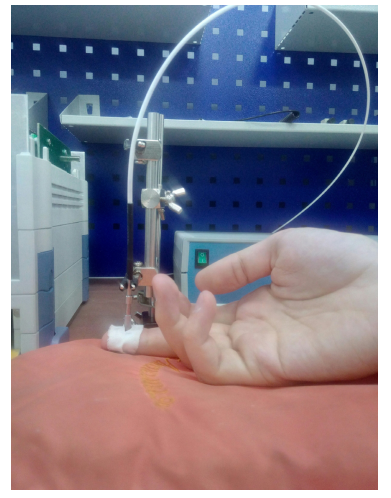

Figure 2. Location of the fiber optic probe during the measurements

It is well-known that one of the most important factors influencing the endogenous fluorescence is the activity of metabolic processes inside the cells. ${ }^{16}$ The metabolic activity in living tissue is connected and mutually interrelated with the intensity of blood microflow. ${ }^{17,18}$ Therefore, in this study brachial arterial occlusion test was applied as a provocative factor to change blood flow in the region of interest. Thus, the study included the registration of diffuse reflection spectra and fluorescence spectra in three stages (Fig. 3). 


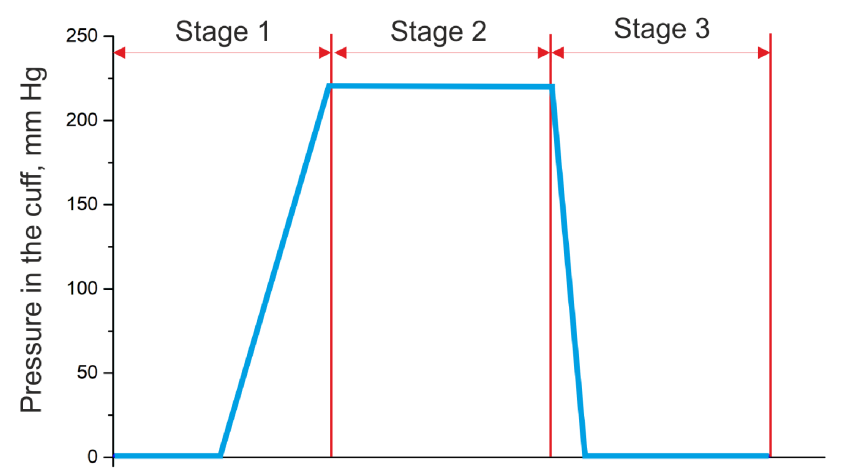

Figure 3. Graph of pressure in the cuff during the applied occlusion test

The first stage included the registration of fluorescence and diffuse reflectance spectra without the use of provocative factor. At the second stage, fluorescence and diffuse reflectance spectra were registered after the arterial occlusion test (the pressure in the cuff reached $220 \mathrm{mmHg}$ ). During the third stage, fluorescence and diffuse reflectance spectra were registered in 30 seconds after the cuff pressure normalization. The collected spectra has been processed in the developed software in order to obtain the compensated fluorescent spectra.

\section{RESULTS AND DISCUSSION}

Compensation of the blood influence was carried out by dividing experimentally obtained FS spectrum on the DRS spectrum. In figures 4, 5 and 6 the experimentally obtained spectra of the DRS and FS and compensated FS are given respectively.
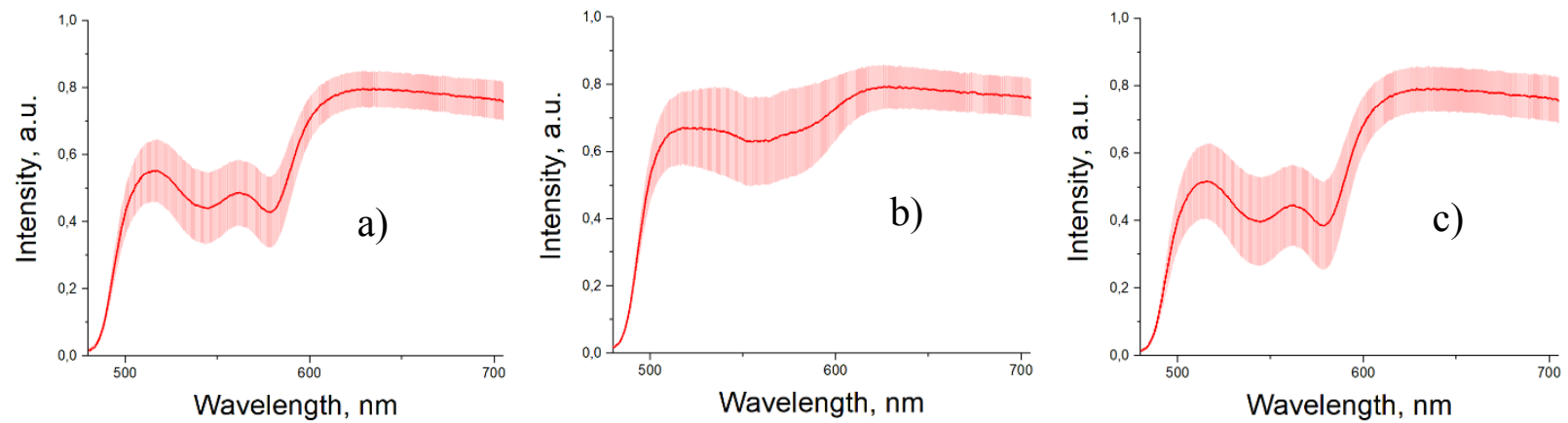

Figure 4. Mean and SD of diffuse reflectance spectra before (a), during (b) and after (c) the occlusion test
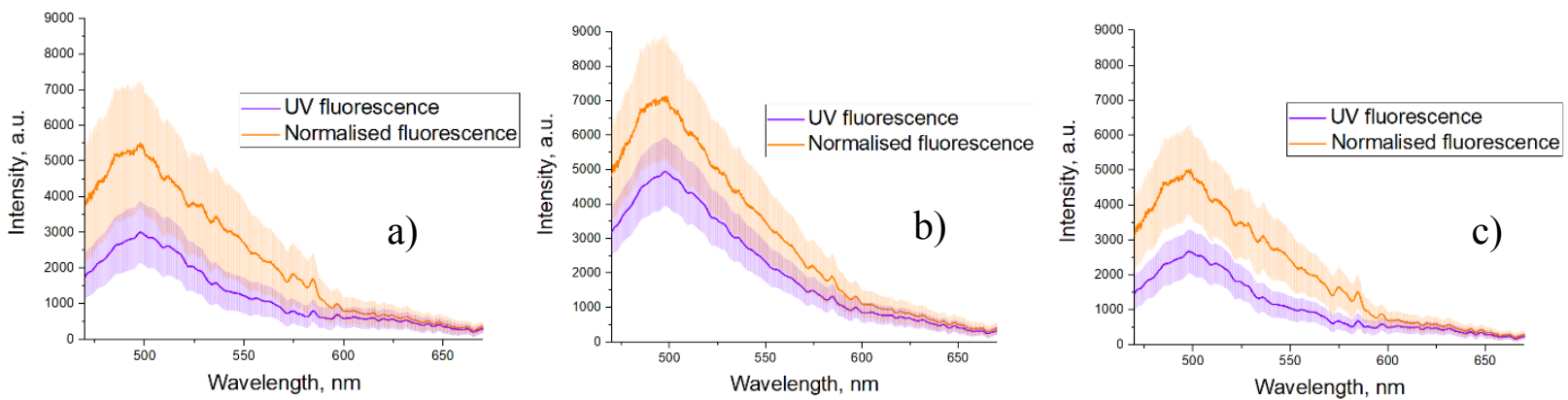

Figure 5. Mean and SD of raw and normalized fluorescence spectra at UV (365 nm) before (a), during (b) and after (c) the occlusion test 

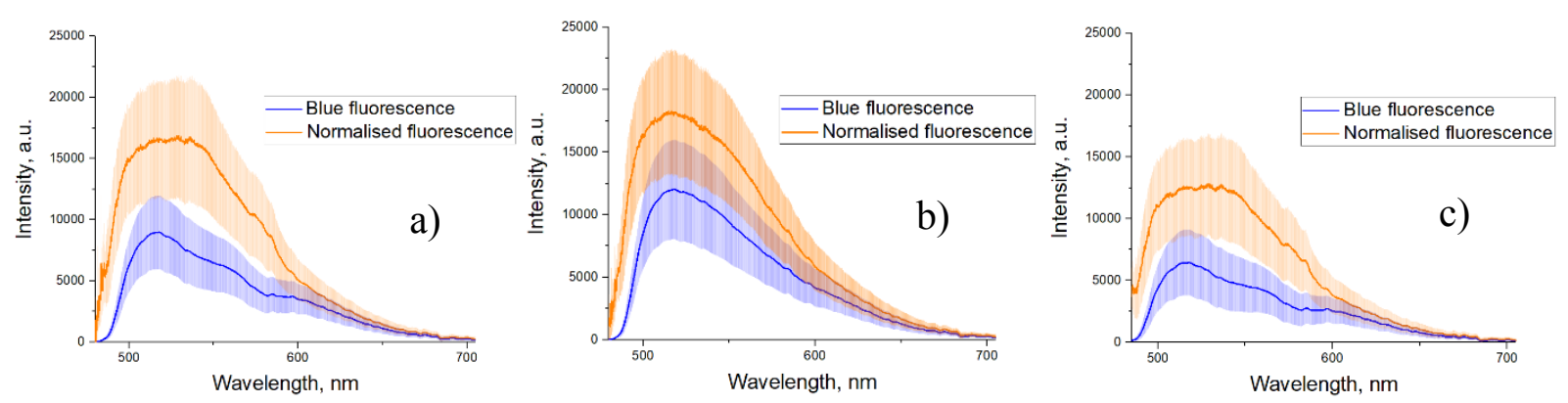

Figure 6. Mean and SD of raw and normalized fluorescence spectra at blue (450 nm) before (a), during (b) and after (c) the occlusion test

The implemented multimodal approach for the in vivo optical measurements in combination with occlusion test for minimisation of the blood influence has shown good repeatability of obtained experimental fluorescence spectra. The conducted experiment has demonstrated that the proposed approach of fluorescence measurements accomplished by simultaneous real-time measurements of diffuse reflectance spectra allows one to increase the repeatability of the measurements presented. It is worth noting that artificial ischemia caused by blood flow termination also affects the intensity of metabolic processes, and hence the fluorescence spectra. This effect can explain the residual difference between the fluorescence spectra at different stages of the occlusion.

\section{CONCLUSIONS}

The obtained results prove the possibility of using the proposed method as a normalizing approach in all areas where FS is applied. Blood compensation is necessary to determine the concentration of different fluorophores inside biological tissue which can be used as essential diagnostic information. The developed setup is rather compact, and the software allows for carrying out measurements in real time that can be applied in the practical optical spectroscopy and potentially in medicine.

\section{ACKNOWLEDGEMENTS}

The work was funded by Russian Foundation for Basic Research according to the research project №18-02-00669.

\section{REFERENCES}

[1] Lakowicz, J. R., [Principles of Fluorescence Spectroscopy], Kluwer Academic Publishers, 954 (2006).

[2] Rogatkin, D. A., "Physical foundations of laser clinical fluorescence spectroscopy in vivo," Medical Physics. 4, 78-96 (2014).

[3] Dunaev A.V., Dremin V.V., Zherebtsov E.A., Rafailov I.E., Litvinova K.S., Palmer S.G., Stewart N.A., Sokolovski S.G., Rafailov E.U., "Individual variability analysis of fluorescence parameters measured in skin with different levels of nutritive blood flow," Medical Engineering \& Physics. 37(6), 574-583 (2015).

[4] Dremin V.V., Dunaev A.V., "How the melanin concentration in the skin affects the fluorescence-spectroscopy signal formation," Journal of Optical Technology. 83(1), 43-48 (2016).

[5] Dremin, V. V, Zherebtsov, E. A., Rafailov, I. E., Vinokurov, A. Y., Novikova, I. N., Zherebtsova, A. I., Litvinova, K. S. and Dunaev, A. V., "The development of attenuation compensation models of fluorescence spectroscopy signals," Saratov Fall Meeting 2015: Third International Symposium on Optics and Biophotonics and Seventh Finnish-Russian Photonics and Laser Symposium. 9917, 99170 (2016).

[6] Jacques, S. L., "Optical properties of biological tissues: a review," Physics in Medicine \& Biology. 58(11), R37-61 (2013).

[7] Bradley, R. S. and Thorniley, M. S., "A review of attenuation correction techniques for tissue fluorescence," J. R. Soc. Interface. 3(6), 1-13 (2006). 
[8] Zherebtsov, E., Dremin, V., Zharkikh, E., Zherebtsova, A., Rafailov, I., Dunaev, A. \& Rafailov, E., "Fibre-optic probe for fluorescence diagnostics with blood influence compensation," Dynamics and Fluctuations in Biomedical Photonics XV. - International Society for Optics and Photonics. 10493, 104931L (2018).

[9] Farrell T. J., Wilson B. C., Patterson M. S., "The use of a neural network to determine tissue optical properties from spatially resolved diffuse reflectance measurements," Physics in Medicine \& Biology. 37(12), 2281 (1992).

[10] Matcher S. J., Cooper C. E., "Absolute quantification of deoxyhaemoglobin concentration in tissue near infrared spectroscopy," Physics in Medicine \& Biology. 39(8), 1295 (1994).

[11] Liu, H., Boas, D. A., Zhang, Y., Yodh, A. G., \& Chance, B., "Determination of optical properties and blood oxygenation in tissue using continuous NIR light," Physics in Medicine \& Biology. 40(11), 1983 (1995).

[12] Tuchin V. V. et al. [Optical biomedical diagnostics], Fizmatlit, Moscow, 77 (2007).

[13]Zherebtsov, E. A., Kandurova, K. Y., Seryogina, E. S., Kozlov, I. O., Dremin, V. V., Zherebtsova, A. I., ... \& Meglinski, I., "The influence of local pressure on evaluation parameters of skin blood perfusion and fluorescence," Saratov Fall Meeting 2016: Optical Technologies in Biophysics and Medicine XVIII. International Society for Optics and Photonics. 10336, 1033608 (2017).

[14]Zherebtsov, E. A., Zherebtsova, A. I., Doronin, A., Dunaev, A. V., Podmasteryev, K. V., Bykov, A. and Meglinski, I., "Combined use of laser Doppler flowmetry and skin thermometry for functional diagnostics of intradermal finger vessels," Journal of biomedical optics. 22(4) (2017).

[15]Zherebtsov, E.; Doronin, A.; Bykov, A.; Popov, A.; Meglinski, I., "Impact of blood volume on the diffuse reflectance spectra of human skin in visible and NIR spectral ranges," European Conference on Biomedical Optics. Optical Society of America. 104120H (2017).

[16]Zherebtsov, E., Angelova, P., Sokolovski, S., Abramov, A., \& Rafailov, E., "Verification of NADH content measurements by portable optical diagnostic system in living brain tissue," Photonic Solutions for Better Health Care VI. International Society for Optics and Photonics. 10685, 106854E (2018).

[17] Krupatkin A.I., Sidorov V.V., Dremin V.V., Dunaev A.V., Novikova I.N., Zhu S.; Nabi G., Litvinova K.S., Baklanova A.P., Bakshaliev R.M., Ravcheev S.A., "Evaluating adaptation options of microcirculatory-tissue systems based on the physiological link of nutritive blood flow and redox ratio," Optical Technologies in Biophysics and Medicine XVI; Laser Physics and Photonics XVI; and Computational Biophysics. 9448, 944803 (2014).

[18] Mayevsky A., Ziv I., "Oscillations of cortical oxidative metabolism and microcirculation in the ischaemic brain," Neurological research. 13(1), 39-47 (1991). 